\title{
Ferromagnetic vortex core switching at elevated temperatures
}

\author{
Kristof M. Lebecki* \\ Department of Physics, University of Konstanz, D-78457 Konstanz, Germany \\ and Nanotechnology Centre, VSB-Technical University of Ostrava, 17 listopadu 15, CZ-708 33 Ostrava, Czech Republic
}

Ulrich Nowak

Department of Physics, University of Konstanz, D-78457 Konstanz, Germany

(Received 3 September 2013; revised manuscript received 27 November 2013; published 23 January 2014)

\begin{abstract}
An approach for the investigation of vortex core switching is presented. Thermal effects up to the Curie point are included in a micromagnetic framework based on the recently developed Landau-Lifshitz-Bloch equation. In this approach it is easier to avoid numerical discretization artifacts, commonly present when a Bloch point is mediating the switching process. Switching in thin circular permalloy disks caused by the application of a slowly increasing magnetic field oriented orthogonally to the disk is considered. An energy barrier which can be overcome by thermal fluctuations is taken into account, leading to a strong influence of the temperature on the switching field. In particular, the switching field goes to zero at a significantly smaller temperature than the Curie temperature. The deduced nucleation volume is smaller than the typical grain size in permalloy.
\end{abstract}

DOI: 10.1103/PhysRevB.89.014421

\section{INTRODUCTION}

A ferromagnetic vortex is mainly an in-plane domain structure with a rotational character except for its very center, where the magnetization points out of plane [1]. This magnetic texture has attracted quite a lot of interest recently since possible applications in magnetic memory devices [2] or gigahertz-frequency generators [3] have been proposed. The central part of the vortex, where the magnetization is orthogonal to the plane is called the vortex core (VC)-see Fig. 1 where we have marked the $\mathrm{VC}$ in blue. It is very thin, usually a few nanometers in diameter. VC switching can happen either in a quasistatic way-e.g., by applying an orthogonal, slowly increasing magnetic field [4-6], or dynamically-e.g., by applying magnetic [7-9] or electric [10] pulses, or oscillating magnetic $[9,11-14]$ or electric $[2,3]$ fields.

In every case, however, VC switching is accompanied by the short presence of a very unstable object - the Bloch point (BP) [7]. The BP is a mathematical singularity-the magnetization cannot be defined at this point within the framework of a continuum theory (micromagnetism) $[15,16]$. This makes the description of VC reversal a very difficult task $[4,17]$. Only at elevated temperature this problem can be avoided, as the function $\mathbf{M}(\mathbf{r})$ becomes continuous [18,19]. (We note in passing that Elias and Verga also use in this context the name "singularity" [18]-this is, however, strictly speaking not correct; for nonzero temperature the magnetization at the BP and in its neighborhood is well defined, and it is also differentiable.) Considering a finite temperature has two additional advantages: First, it is more realistic since the temperature is finite in every experiment. Second, there has been a clear interest recently in temperature-assisted or temperature-mediated reversal phenomena [20,21].

In this paper we extend our latest study [19] revealing properties of the BP and VC reversal. We consider a flat

*Lebecki@fuw.edu.pl
PACS number(s): 75.78.Cd, 75.60.Jk, 75.60.Nt, 75.75.Fk circular sample made of soft permalloy containing a magnetic vortex. A magnetic field oriented antiparallel to the vortex core is applied, slowly increasing. Then the process of VC switching is carefully monitored. We pay special attention to "discretization mesh artifacts" since it is well known that the introduction of numerical cells strongly influences BP-related phenomena $[4,19,22]$. In our analysis we check a wide range of discretization grids and extrapolate our results towards mesh independence.

\section{METHODS}

We follow a temperature-dependent, numerical, micromagnetic approach, based on the Landau-Lifshitz-Bloch (LLB) equation of motion [23]. The LLB equation is an extension of the well-known Landau-Lifshitz-Gilbert (LLG) equation of motion [24,25] for the case of nonzero temperature, $T \geqslant 0$. It can be written in the form [23]

$$
\begin{aligned}
\dot{\mathbf{M}}= & -\bar{\gamma} \mathbf{M} \times \mathbf{H}_{\mathrm{eff}}+\bar{\gamma} \alpha_{\|} \frac{M_{\mathrm{s}}}{M^{2}}\left(\mathbf{M} \cdot \mathbf{H}_{\mathrm{eff}}\right) \mathbf{M} \\
& +\bar{\gamma} \alpha_{\perp} \frac{M_{\mathrm{s}}}{M^{2}}\left(\mathbf{M} \times \mathbf{H}_{\mathrm{eff}}\right) \times \mathbf{M} .
\end{aligned}
$$

Here, $\bar{\gamma}$ is the gyromagnetic ratio, $\mathbf{M}$ is the magnetization, $\mathbf{H}_{\mathrm{eff}}$ is the effective field [see Eq. (2)], $\alpha_{\|}$and $\alpha_{\perp}$ are respectively longitudinal and transversal damping coefficients [see Eq. (3)], $M_{\mathrm{s}}$ is the saturation magnetization at $T=0$, and $M=|\mathbf{M}|$. The effective field is similar to that in the case of the LLG equation but with an additional term that controls the deviation of the magnetization magnitude from its equilibrium value $M_{\mathrm{e}}(T)$,

$$
\mathbf{H}_{\mathrm{eff}}=\mathbf{H}_{\mathrm{d}}+\mathbf{H}_{\mathrm{ext}}+\frac{2 A \nabla^{2} \mathbf{M}}{\mu_{0} M_{\mathrm{e}}^{2}}-\left(\frac{M^{2}}{M_{\mathrm{e}}^{2}}-1\right) \frac{\mathbf{M}}{2 \chi_{\|}},
$$

where $\mathbf{H}_{\mathrm{d}}$ and $\mathbf{H}_{\mathrm{ext}}$ are respectively the demagnetization and the external field, $A$ is the exchange constant, $\mu_{0}$ is the vacuum permeability, and $\chi_{\|}$is the longitudinal susceptibility. We ignore here anisotropy effects as we focus our attention on permalloy. Of course we have $M_{\mathrm{e}}(0)=M_{\mathrm{s}}$. The longitudinal 


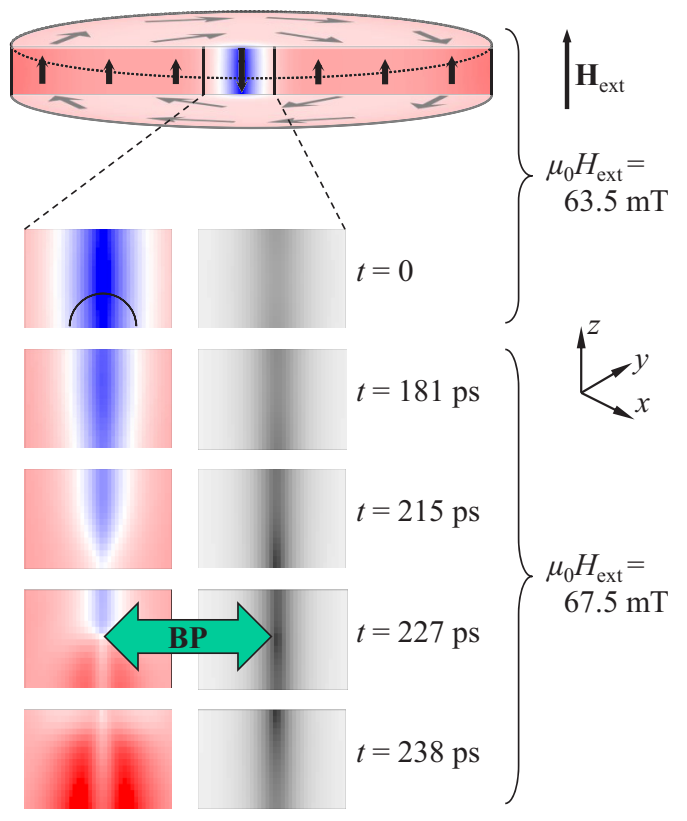

FIG. 1. (Color online) Upper figure: Sketch of the numerical experiment. The magnetic field is applied perpendicular to the diskshaped sample containing a vortex structure. The blue-colored VC is pointing downwards ( $-z$ direction), and the field is applied upwards. As the field is too small to switch the VC, the structure of the vortex is modified with a visible $+z$ component of the magnetization due to the applied external field. (The magnetization component $M_{z}$ slightly increases when the disk edge is approached.) Still, the rotational structure of the vortex is retained. The color coding describes here the magnetization component along the $z$ axis. Lower figures: Snapshots presenting the enlarged $x z$ cross sections of the vortex core vicinity for two fields: just before the core switches and during this process. After some time the structure starts to change: the BP (marked with an arrow) is traveling along the VC, leading to its reversal. Figures on the left have the same color coding as the top figure. Pictures on the right have a color coding representing the magnetization magnitude, where white means $M(\mathbf{r})=M_{\mathrm{e}}$ and black means $M(\mathbf{r})=$ 0 . After the structure relaxes at $\mu_{0} H_{\text {ext }}=67.5 \mathrm{mT}$ (not shown here), appropriate snapshots would be, respectively, uniformly dark red and uniformly white. $T=850 \mathrm{~K}$; the half circle in the upper snapshot shows the nucleation half sphere $V_{\mathrm{n}}$ (see text for details).

and transversal damping coefficients are given by

$$
\alpha_{\|}=\alpha 2 T / 3 T_{\mathrm{C}}, \quad \alpha_{\perp}=\alpha\left(1-T / 3 T_{\mathrm{C}}\right),
$$

where $\alpha$ is the Gilbert damping constant at the atomic level, $T_{\mathrm{C}}$ is the Curie temperature, and only the case $T \leqslant T_{\mathrm{C}}$ is considered [23].

In contrast to the zero-temperature LLG equation, in the LLB approach the material parameters are actually temperature-dependent functions, namely, $M_{\mathrm{e}}(T), A(T)$, and $\chi_{\|}(T)$. These functions must be provided as input for the modeling process, the details of which can be found elsewhere $[19,26]$. Another important feature of the LLB equation is the nonconstant magnetization magnitude, as $M(\mathbf{r}, t)$ can vary in space and in time. As already mentioned, this process is monitored by the last term in Eq. (2). Thus, for small temperatures, where $\chi_{\|}$is small, one can expect $M$ to be close to its equilibrium value, while for larger temperatures, where $\chi_{\|}$increases (diverging at $T_{\mathrm{C}}$ ), local differences between $M$ and $M_{\mathrm{e}}(T)$ can be larger. Corresponding magnetization drops can be observed especially inside a domain wall [27] or inside a VC [26]. In the vicinity of a BP (a zero-dimensional object) the magnetization drop is even more pronounced: for any finite temperature $T>0$ the magnetization goes to zero here $[19,28]$ although, as we will show in the following, in numerical simulations this tendency is limited by the introduced discretization.

Our code is an extension of the well-established simulation package OOMMF [29]. In our implementation we have followed Ref. [30] apart from omitting the thermal stochastic field. Instead, we consider thermal activation by calculating the energy barrier that appears during the BP-related reversal. This approach has the advantage that we can take into account time scales that are much longer than usually available in micromagnetic modeling. To compare with experiment we have selected an escape time of $1 \mathrm{~min}$ so that the energy barrier that has to be overcome by thermal activations is $\Delta E_{\text {ther }}(T)=25 k_{\mathrm{B}} T$.

The sample we have modeled was a thin ferromagnetic disk, with a diameter of $200 \mathrm{~nm}$ and a thickness of $20 \mathrm{~nm}$. Material parameters were chosen to mimic permalloy: $A(0)=$ $13 \times 10^{-12} \mathrm{~J} / \mathrm{m}, M_{\mathrm{e}}(0)=0.86 \times 10^{6} \mathrm{~A} / \mathrm{m}$, and $T_{\mathrm{C}}=870 \mathrm{~K}$. We have neglected anisotropy effects. The damping constant $\alpha$ was 0.01 , but we have made also comparative simulations for $\alpha=0.5$. Initially we prepared our sample with a vortex state in its center. Then the external field $H_{\text {ext }}$ was applied approximately orthogonally to the sample (antiparallel to the VC) but slightly tilted by ca. $1^{\circ}$ away from the $z$ axis (see the sketch Fig. 1). $H_{\text {ext }}$ was increased in small steps (step size smaller than or equal to $20 \mathrm{mT}$ ) until it reached the critical value $H_{\mathrm{sw}}$ where the vortex core switched. Figure 1 shows snapshots of the sample taken at times just before, during, and at the end of the VC reversal $\left(63.5 \leqslant \mu_{0} H_{\mathrm{sw}} \leqslant 67.5 \mathrm{mT}\right)$.

In micromagnetic simulations one has to introduce a discretization mesh [25]. The cell size is a critical parameter for micromagnetic simulations and so we have conducted a series of numerical experiments for four different grids as listed in Table I. If not specified otherwise results for the finest lattice, grid 1 , are presented. The parameter that describes the quality (and validity) of micromagnetic simulations is the maximal angle between magnetization vectors in neighboring cells. For simulations with variable magnetization we have proposed to replace this by the relative maximal magnetization change between neighboring cells, $\Delta m_{\max }$ [19]. In thin structures $\Delta m_{\max }$ is often more influenced by the in-plane cell size. This is the reason why we could use a larger cell $z$ edge, $c_{z}$, as compared to the in-plane cell edges, $c_{x y} \stackrel{\text { def }}{=} c_{x}=c_{y}$ (see

TABLE I. List of mesh grids used. Every cell was a rectangular prism with dimensions $c_{x} \times c_{y} \times c_{z}$.

\begin{tabular}{lccc}
\hline \hline Grid number & $c_{x}(\mathrm{~nm})$ & $c_{y}(\mathrm{~nm})$ & $c_{z}(\mathrm{~nm})$ \\
\hline 1 & 0.78125 & 0.78125 & 1.25 \\
2 & 1.5625 & 1.5625 & 2 \\
3 & 2 & 2 & 2.5 \\
4 & 2.5 & 2.5 & 2.5 \\
\hline \hline
\end{tabular}




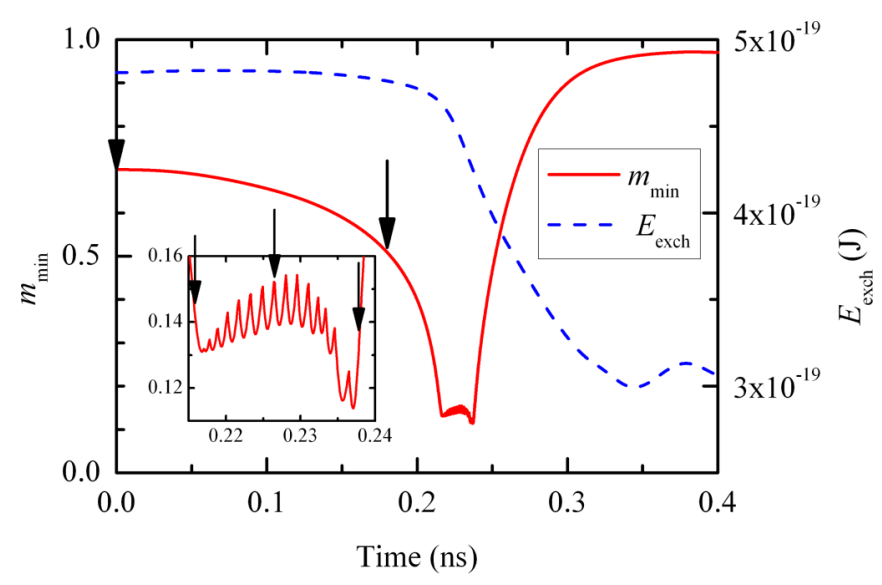

FIG. 2. (Color online) Normalized minimal magnetization length found across all cells, $m_{\min }$, as a function of time (left axis). Right axis shows the exchange energy $E_{\text {exch }}$. We present here data for a short time period just after having increased the field from 63.5 to $67.5 \mathrm{mT}$ as shown in Fig. 1. Inset: $m_{\min }(t)$ while the BP crosses the sample. The snapshots in Fig. 1 were taken at the times marked with arrows.

Table I) and still our $\Delta m_{\max }$ was larger along the $x$ or $y$ direction as compared to changes along the $z$ direction. Thus, in the following we will plot the cell-size dependence of any accuracy versus $c_{x y}$ rather than $c_{z}$.

\section{SWITCHING FIELD: CELL-SIZE DEPENDENCE}

The behavior shown in Fig. 1 is representative for all temperatures we have investigated. In this figure we present, with different color coding (i) the $z$ component of the magnetization (snapshots on the left) and (ii) the magnitude of the magnetization (snapshots on the right). When the external field is applied the vortex core becomes thinner, as reported earlier in Ref. [11]. For $H_{\text {ext }}=63.5 \mathrm{mT}$ the $\mathrm{VC}$ radius is a few times smaller as compared to $H_{\text {ext }}=0$. Another consequence of the applied field is the squeezing of the magnetization length in the VC. Without the field, this squeezing is nearly negligible [26]. Upon further increase in the field the following process happens, where special attention is paid to the magnetization magnitude:

(1) The squeezing of the VC continues. In parallel, the magnetization magnitude in the $\mathrm{VC}$ drops. The latter process can be observed in more detail in Fig. 2, where the minimum of the magnetization lengths of all the cells in the sample is plotted, $m_{\min }(t)$. This happens on a time scale of a few hundred picoseconds (time range 0 to $215 \mathrm{ps}$ in Fig. 1).

(2) The BP is nucleated at one end of the VC and the $\mathrm{BP}$ moves along the $z$ axis, leading to a reversal of the core. According to theory, in the vicinity of the BP $M(\mathbf{r})$ drops to zero [18], but in micromagnetic simulations this trend is limited by the introduced discretization [19]. This happens on a time scale of a few picoseconds (here 215 to $238 \mathrm{ps}$ ).

(3) The whole structure relaxes, having the VC now aligned with the field. The magnetization magnitude inside the VC increases, reaching almost its equilibrium value. This happens on a time scale of a few nanoseconds (here 0.24 to $2.64 \mathrm{~ns}$ ).
The time dependence of the minimal magnetization length found across the sample, $m_{\min }(t)$, is plotted in Fig. 2 together with the exchange energy of the system. As described in the literature, the BP-mediated VC reversal is indeed accomplished by a rapid change of the exchange energy [4,7,22]. Figure 2 shows, however, that $m_{\min }$ can be a handier parameter to observe the switching process. [Another parameter used in this context is $\max (d m / d t)$; cf. Fig. 8 in Ref. [4]].

As already mentioned, according to theory $m_{\min }$ should be zero-as long as $T>0$. The reason why $m_{\min }$ actually has a finite value is related to the discretization. To understand this, let us consider a BP that has, at a given time, its center at point $\mathbf{r}_{0}$. This point belongs to a cell $V$ where the BP can also be on the surface of this cell. Although indeed $M\left(\mathbf{r}_{0}\right)=0$, in finite-difference micromagnetic simulations one actually computes space averages of the magnetization components for the given cell [25]:

$$
M_{i, V}=\int_{V} M_{i}(\mathbf{r}) d^{3} \mathbf{r}, \quad i=(x, y, z) .
$$

If one defines the magnetization length as

$$
M_{V}=\sqrt{M_{x, V}^{2}+M_{y, V}^{2}+M_{z, V}^{2}},
$$

it is obvious that $M_{V}>0$ as long as the cell has a finite volume. A too small $m_{\min }$ is, however, energetically unfavorable due to the last term of Eq. (2), unless $\chi_{\|}$is large, which is true only for temperatures close to $T_{\mathrm{C}}$. Thus, while the BP moves it tries to maximize $m_{\min }$. This leads to the phenomenon-described already in the literature - that the "BP prefers to maximize its distance from mesh points" [4]. When plotting $m_{\min }(t)$ this effect leads to oscillations shown in the inset of Fig. 2 (see also Ref. [4], especially Figs. 8 and 10 there). We note that although these oscillations are caused by an artificial pinning by the lattice of discretization cells, one cannot exclude a similar phenomenon in real samples caused by the lattice of atoms.

We would like to stress that the parameter $m_{\min }$ is strongly cell-size dependent as long as BP-related processes are present. In the limit of zero cell size, $c \rightarrow 0, m_{\min }(c)$ goes to zero as long as a BP exists. Thus, the limits of small and large times in Fig. 2 where no BP exists are only weakly cell-size dependent. In contrast, while the BP moves through the disk (the central part of Fig. 2) there is a strong cell-size dependence, which is not shown here.

Figure 3(a) shows the switching fields as a function of the temperature summarized for all considered grids (dashed-line-connected points). As already described in the literature, a strong cell-size dependence exists, which we attribute to too large values of the quality parameter $\Delta m_{\max }$. Only results for the temperatures in the range $850-870 \mathrm{~K}$ and for the finest grids fulfill the condition $\Delta m_{\max } \lesssim 1 / 2$ proposed in Ref. [19]. Indeed, if that condition is fulfilled, then the cell-size dependence of the results vanishes. This is another signature supporting the statement that reliable micromagnetic simulations should pass the $\Delta m_{\max }$ test (or its angular formulation in the case of $T=0$ ) [19]. So far, thermal activation which is important in cases where slow processes are considered, like the quasistatic applied field, was excluded from our investigation. We will address these two issues in Sec. V. 

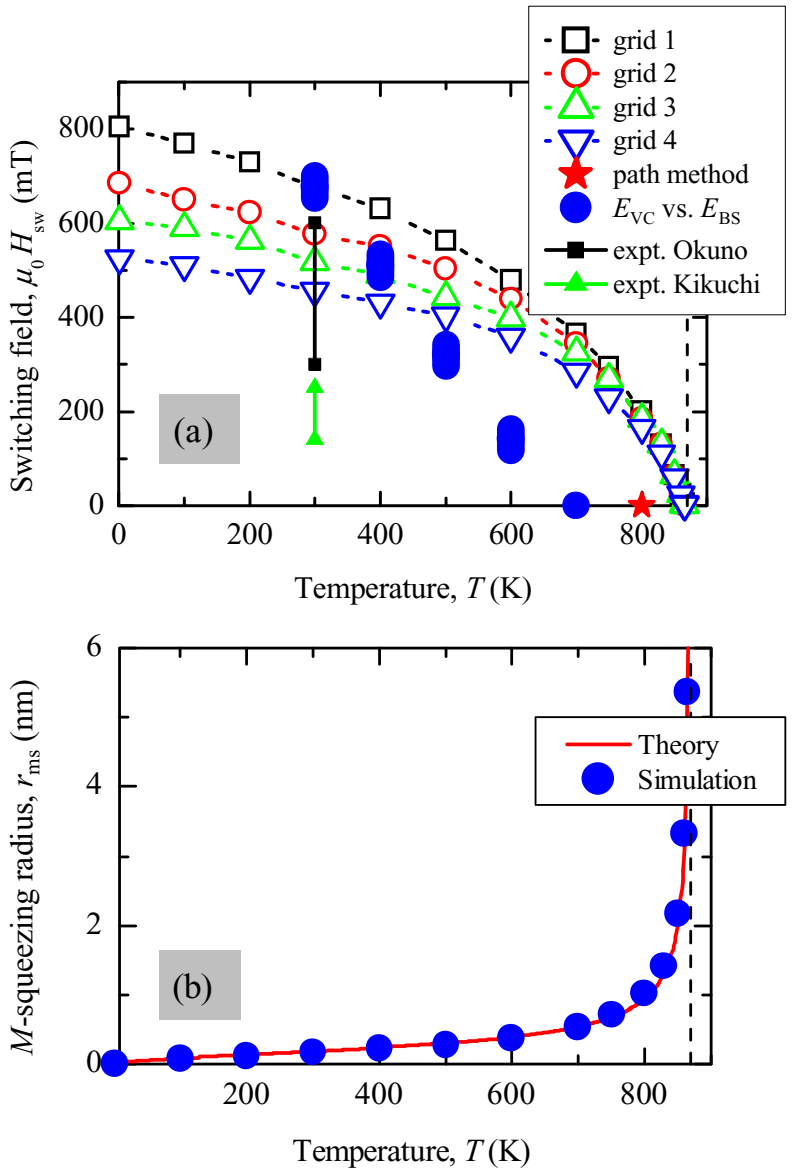

FIG. 3. (Color online) (a) Switching field as a function of temperature. Dashed-line-connected points: results for different mesh grids; see Table I. Solid points: cell-independent results with temperature activation processes included; see Sec. V. Solid-line-connected points: experiments [4,5]. In experiments an array of islands is measured, and thus one obtains a spectrum of values represented in this figure by intervals. (b) Radius of the $M$-squeezing effect, $r_{\mathrm{ms}}$. Values from our simulations (points) are compared with theory (line) $[19,28]$. The vertical dashed line marks $T_{\mathrm{C}}$.

\section{BLOCH POINT GEOMETRY}

Prior work [18] predicts the magnetization magnitude to drop in the vicinity of the BP. In our last paper we confirmed this numerically in the close vicinity of a static BP [19]. Our present simulation enabled us to investigate this effect for a much more physical case-a dynamic BP during switching of a VC. Figure 4 shows cross sections of a central part of our sample, where $M$ for every cell is plotted (points). These data are from a snapshot ( $t=226 \mathrm{ps}$ ), where the BP is roughly in the middle of the sample. We have fitted these data with the following function, introduced already in Ref. [19] and being a handy analytical approximation to the theoretical expected dependence [28],

$$
\begin{aligned}
M(x, y, z) & =M_{\mathrm{e}} \frac{s^{3}+0.827 s^{2}+1.371 s}{s^{3}+0.827 s^{2}+2.371 s+2 \times 1.371}, \\
s & =\frac{\sqrt{\left(x-x_{0}\right)^{2}+\left(y-y_{0}\right)^{2}+\left(z-z_{0}\right)^{2}}}{r_{\mathrm{ms}}} .
\end{aligned}
$$

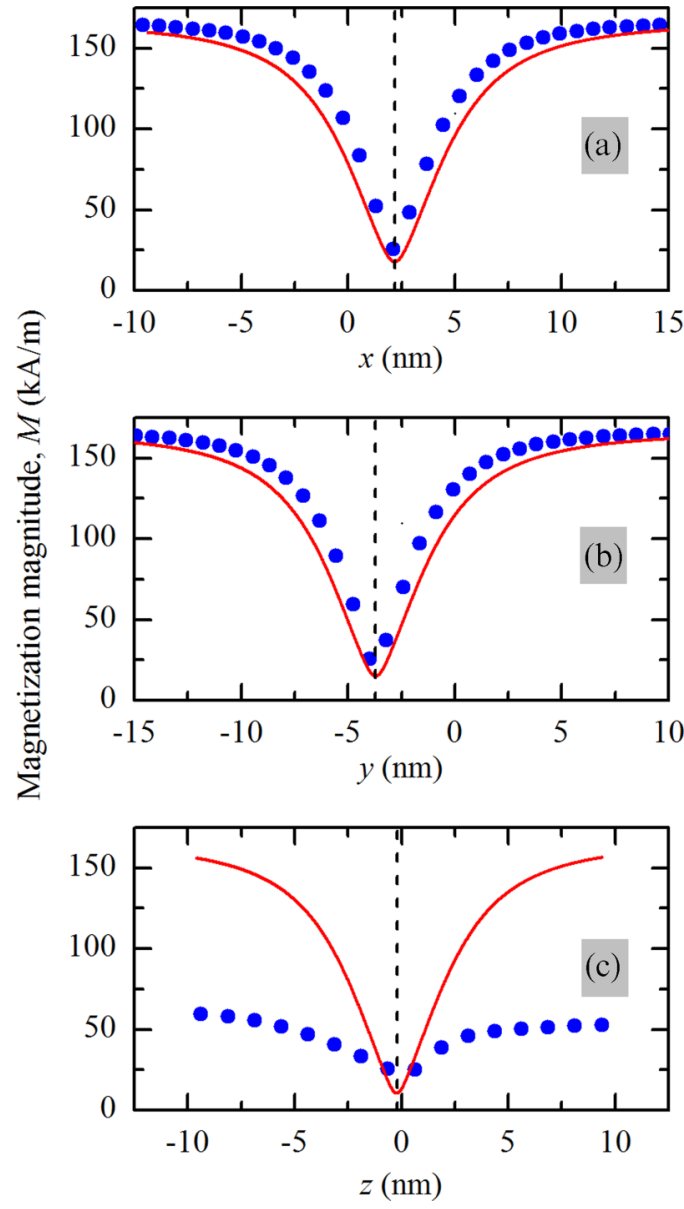

FIG. 4. (Color online) Cross sections through the sample containing a Bloch point (points). The magnitude of the magnetization vector is plotted as a function of the position. Lines indicate the fitted curve following Eq. (6). Dashed lines mark the BP. The origin of our coordinate system is placed in the center of the simulated disk. $T=850 \mathrm{~K}$ and $t=226 \mathrm{ps}$ (see Fig. 1).

Here $\mathbf{r}_{0}=\left(x_{0}, y_{0}, z_{0}\right)$ is the position of the BP and $r_{\mathrm{ms}}$ is a scaling factor defining the distance where the magnetization drop is roughly $50 \%$. The fitted function is shown with lines in Fig. 4. The fitting parameters are $x_{0}, y_{0}, z_{0}$, and $r_{\mathrm{ms}}$. For cross sections along the $x$ and $y$ axes this function agrees quite well with our simulations. This is not the case for the cross section along the $z$ axis. Here, the BP-related " $M$-squeezing effect" [19] overlaps with the VC-related one, and thus the overall magnetization drop is more pronounced. Additionally, an asymmetry between cases with $z<z_{0}$ (VC not yet switched) and $z>z_{0}$ (VC already switched) can be seen. The reason why the cross sections in Fig. 4 do not go to zero close to the $\mathrm{BP}(s \ll 1)$ [see Eq. (6)], is quite simple: the "BP prefers to sit farthest from mesh points" [4] and thus $x_{0}, y_{0}$, and $z_{0}$ do not coincide with mesh points while the cross sections shown in Fig. 4 are selected according to the mesh points.

The parameter $r_{\mathrm{ms}}$ as returned by our fitting procedure for all considered temperatures is shown in Fig. 3(b). For comparison we also show the theoretically predicted function $[19,28]$

$$
r_{\mathrm{ms}, \text { theory }}(T)=2 \sqrt{\chi_{\|}(T) A(T) / \mu_{0} M_{\mathrm{e}}(T)^{2}} .
$$


Good agreement can be observed even for small temperatures. The results presented in Fig. 3(b) depend only weakly on the chosen time (226 ps in the case of Fig. 4). There is indeed some monotonic change in the $r_{\mathrm{ms}}$ as the Bloch point rushes through the sample, with $r_{\mathrm{ms}}$ decreasing with time. This driftalthough larger than the $r_{\text {ms }}$ fitting errors - is, however, a small effect and smaller than the points plotted in Fig. 3(b).

As already mentioned $r_{\mathrm{ms}}$ defines a volume surrounding the $\mathrm{BP}$, where the magnetization squeezing is remarkable. It is a handy measure of this effect. Note, however, that according to our simulations this volume has an elongated shape, stretched in the VC direction. Also, some asymmetry along the $z$ axis is present; see Fig. 4(c).

\section{INCLUSION OF THERMAL EXCITATIONS}

\section{A. Zero applied field}

To include thermal activation effects that affect the switching process we have followed two different approaches. The first one is related to the path method described in Ref. [31]. In this approach the energy landscape is evaluated, different paths connecting the initial and the final state are investigated, and the (separating) energy barrier is determined. The application of this method to an investigation of $\mathrm{VC}$ reversal was already shown by Thiaville et al. [4]. Here we follow this idea for the case of $H_{\text {ext }}=0$, where the energy barrier is easier to calculate. We consider three states:

(1) The VC is not yet switched.

(2) The BP is exactly in the middle of the sample.

(3) The VC is already switched.

The first and the last states have the same energy $E_{1}=$ $E_{3}=E_{\text {min }}$. The second state has the highest possible energy $E_{\max }$. Thus, the energy barrier that has to be overcome is [4]

$$
\Delta E=E_{\max }-E_{\min } .
$$

We have calculated $E_{\max }$ by simulating the $\mathrm{BP}$ that is artificially pinned by the discretization lattice [4,19]. This approach works only for $H_{\text {ext }}=0$ as otherwise the pinning force is too small. The resulting values for $\Delta E$ are shown in Fig. 5 for different grids and different temperatures and compared to the thermal activation threshold $\Delta E_{\text {ther }}(T)=$ $25 k_{\mathrm{B}} T$. The VC will be thermally switched if $\Delta E_{\text {ther }}>\Delta E$, so that we identified the intersections of the line $\Delta E_{\text {ther }}(T)$ with linear interpolations for the corresponding $\Delta E$ data and plotted them in Fig. 6 (left axis). We did this for different grids and we have put $c_{x y}$ on the horizontal axis, because this is the limiting factor of our accuracy, as explained earlier in the text. A saturation tendency in this figure is clearly visible, and we have fitted these points with a parabola, thus obtaining the switching temperature for the limit of zero mesh size, $T_{\mathrm{sw}}\left(H_{\mathrm{sw}}=0, c_{x y}=0\right)=808 \pm 2 \mathrm{~K}$.

We note that this thermal switching of the VC resembles very much the superparamagnetic behavior found in small, homogeneously magnetized particles. From this perspective, $T_{\text {sw }}$ could as well be called a "blocking temperature."

\section{B. Nonzero applied field}

To investigate the case of nonzero external field we have followed another idea proposed by Thiaville et al. [4]. The

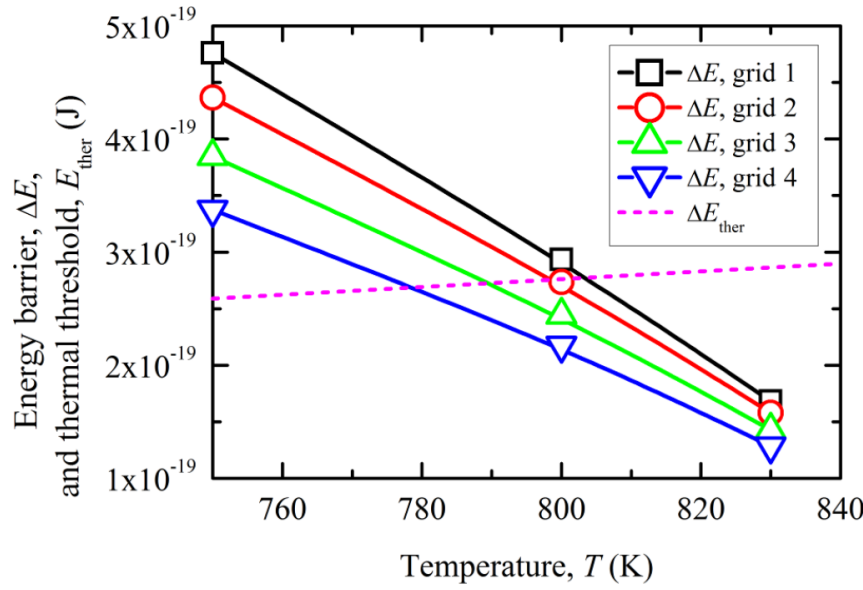

FIG. 5. (Color online) Energy barrier limiting the vortex core switching, $\Delta E$. In the same figure we show the thermal energy $\Delta E_{\text {ther }}=25 k_{\mathrm{B}} T . H_{\mathrm{ext}}=0$.

necessary condition for the BP to enter our sample is that it will lower the energy locally. This condition is weakened by thermal activations, but we will consider it as well. The nucleation starts in the so-called activation (or nucleation) volume $V_{\mathrm{n}}$ [32]. Because we will vary the volume to check for appropriate nucleation conditions, it is convenient to work with the energy density. The energy of the nucleation volume is straightforward to calculate for local or short-range interactions. Thus in the following analysis we will skip dipolar interactions since the considered volumes are small and the demagnetization energy plays a minor role. The energy density

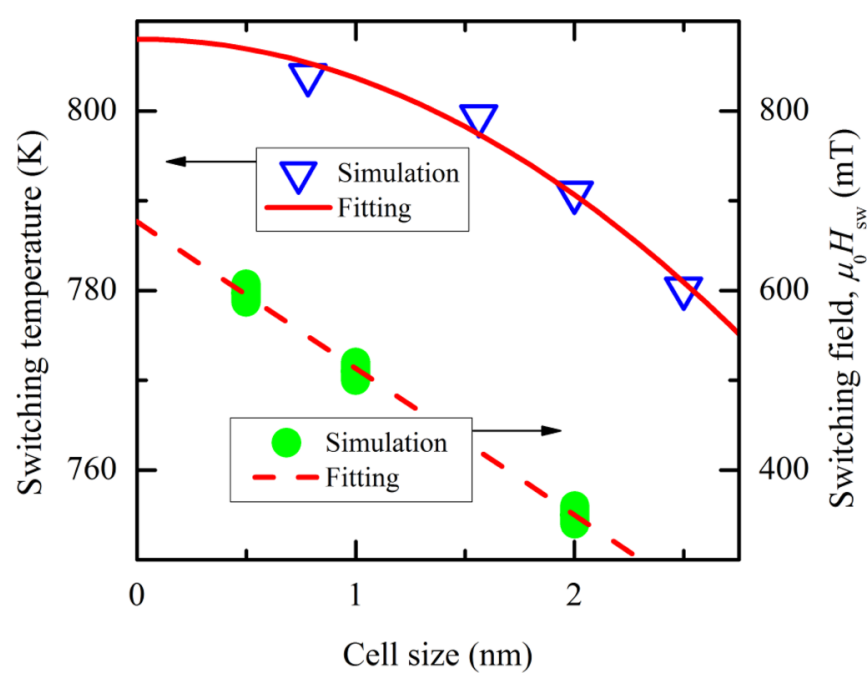

FIG. 6. (Color online) Left axis: Vortex core switching temperature $T_{\mathrm{sw}}$ as a function of the grid (points). The line shows a fit; the limit of small cell size is $T_{\mathrm{sw}}\left(c_{x y}=0\right) \approx 808 \mathrm{~K} . H_{\mathrm{ext}}=0$. Right axis: Switching fields for different cell sizes extracted from Fig. 7(b) (points). Data contain error bars due to the finite $H_{\mathrm{ext}}$ steps. The resulting extrapolation of the linear fit (dashed line) is $\mu_{0} H_{\mathrm{sw}}(c=0) \approx 677 \mathrm{mT} . T=300 \mathrm{~K}$. 

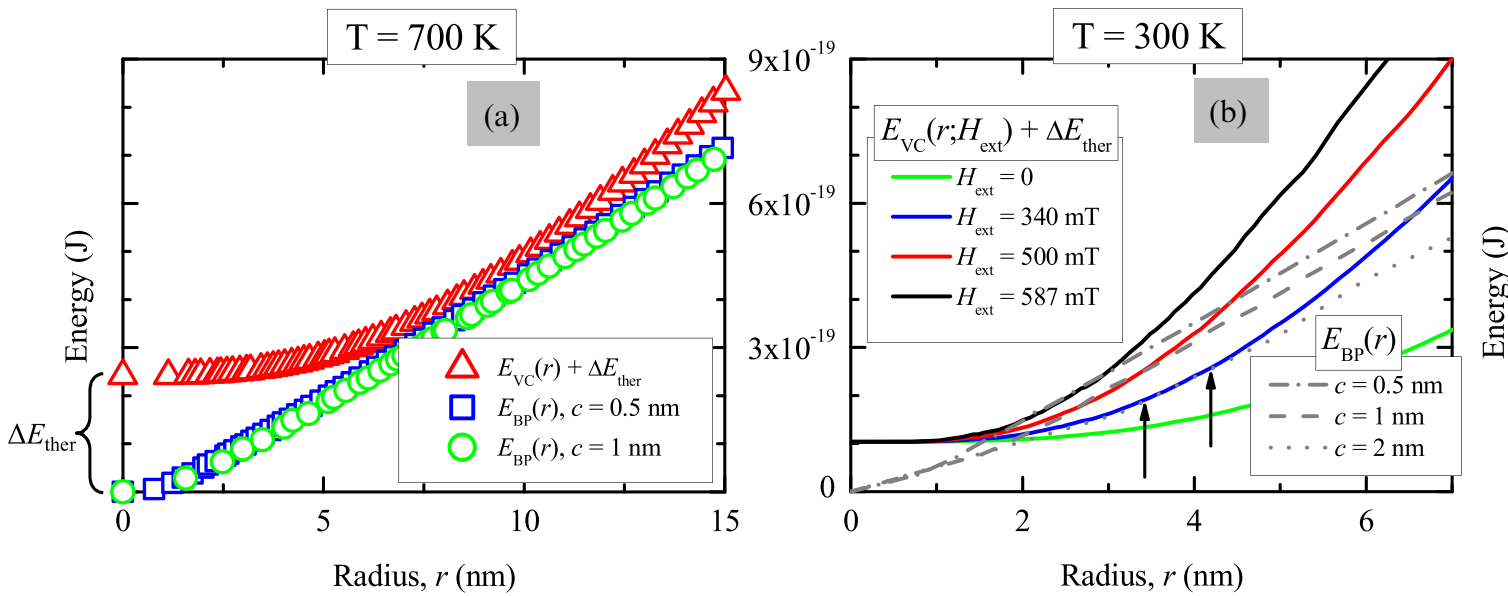

FIG. 7. (Color online) (a) $E_{\mathrm{VC}}$, the radius-dependent energy of a half sphere placed on the sample surface as shown schematically in Fig. 1 . For comparison we present similar results for a sample containing a BP placed at the sample surface, $E_{\mathrm{BP}}$. $E_{\mathrm{VC}}$ is shifted by the thermal activation barrier. As one can see, in the whole radius range the condition $E_{\mathrm{VC}}(r)+\Delta E_{\mathrm{ther}}>E_{\mathrm{BP}}(r)$ is fulfilled. This means that in this case the Bloch point will be thermally activated. $H_{\mathrm{ext}}=0$ and $T=700 \mathrm{~K}$. (b) The same, now for $T=300 \mathrm{~K}$. Because of the large number of curves we have connected the data with points here. Solid, colored lines describe $E_{\mathrm{VC}}$. The dependence of the applied field is clear: the higher $H_{\text {ext }}$, the steeper the curves. Gray, noncontinuous lines describe $E_{\mathrm{BP}}$ for different grid cell sizes $c$. Selected $H_{\text {ext }}$ represent the switching fields for appropriate grid sizes. Arrows show how the nucleation radius was retrieved-by evaluating the crossing of appropriate curves, here $E_{\mathrm{VC}}\left(H_{\mathrm{ext}}=340 \mathrm{mT}\right)$ and $E_{\mathrm{BP}}(c=2 \mathrm{~nm})$.

for the exchange interactions is given by [25]

$$
e_{\mathrm{ex}}(\mathbf{r})=\frac{A}{M_{\mathrm{e}}^{2}}\left\{\left[\nabla M_{\mathrm{x}}(\mathbf{r})\right]^{2}+\left[\nabla M_{\mathrm{y}}(\mathbf{r})\right]^{2}+\left[\nabla M_{z}(\mathbf{r})\right]^{2}\right\} .
$$

We have also considered the energy related to the last term in Eq. (2). The associated energy density has the form of the Landau expansion:

$$
e_{\mathrm{L}}(\mathbf{r})=\frac{\mu_{0}}{8 \chi_{\|} M_{\mathrm{e}}^{2}}\left[M_{\mathrm{e}}^{2}-M^{2}(\mathbf{r})\right]^{2} .
$$

Following the method of Thiaville et al., we have summed up these two components for $V_{\mathrm{n}}$ with the assumption that $V_{\mathrm{n}}$ is a half sphere centered on the sample surface in accordance with the VC axis-i.e., the point where the nucleation potentially happens [4]. $V_{\mathrm{n}}$ has the radius $r$ which is indicated schematically in Fig. 1. The energy of $V_{\mathrm{n}}$ is

$$
E_{\mathrm{VC}}(r)=\int_{V_{\mathrm{n}}(r)}\left[e_{\mathrm{ex}}(\mathbf{r})+e_{\mathrm{L}}(\mathbf{r})\right] d^{3} \mathbf{r} .
$$

The exact position of the half-sphere center was obtained by fitting the $z$ magnetization component at the surface with a two-dimensional Gaussian function.

We have calculated $E_{\mathrm{VC}}(r)$ by numerically interpreting Eq. (9) in every considered case. Due to space discretization, one cannot get a smooth result for $E_{\mathrm{VC}}(r)$ for low radii since here the data are more sparse [see Fig. 7(a)]. The $E_{\mathrm{VC}}(r)$ profile can be compared with the profile of a hypothetical BP placed at the same point. The BP structure is complex and there are no reports about its geometry for the case of an applied external field. But there are reports about the BP structure for the case of $H_{\text {ext }}=0$; see, e.g., Ref. [18]. In our previous paper we also discussed this issue for the case $T>0$ [19]. Of course, application of the field affects the structure of the BP, but this effect is stronger far away from the BP, while close to it exchange interactions are dominant as compared to the
Zeeman interaction. We thus assume that for small $r$ one can approximate the half-sphere energy profile (strictly speaking, the exchange and Landau expansion terms of the energy) of a $\mathrm{BP}$ entering the sample with a dependence $E_{\mathrm{BP}}(r)$ containing appropriate energy terms extracted from simulations with a static BP, as described in Ref. [19]. The resulting $E_{\mathrm{VC}}(r)$ and $E_{\mathrm{BP}}(r)$ profiles are plotted in Fig. 7. As already reported, $E_{\mathrm{BP}}(r)$ has roughly a linear dependence (except for small $r$ ), while $E_{\mathrm{VC}}(r)$ has a parabolalike dependence [4]. We obtained $E_{\mathrm{BP}}$ data for three different cell sizes, where the cells were cubes with edge sizes $c$ equal to $0.5,1$, and $2 \mathrm{~nm}$. While analyzing the Bloch point the cell-size dependence is very important-especially at smaller temperatures; cf. Figs. 7(a) and 7(b) [19]. Taking thermal activation into account, nucleation will set in when [4]

$$
E_{\mathrm{VC}}(r)+\Delta E_{\text {ther }}>E_{\mathrm{BP}}(r) .
$$

From Fig. 7(a) one can see that already for $T=700 \mathrm{~K}$ this condition is fulfilled, in contrast to the more precise result of the path method (ca. $800 \mathrm{~K}$ ). We will comment on this discrepancy later in the text.

We have applied the above approach to different temperatures. Figure 7(b) shows the case of room temperature. As described earlier [4], the VC is squeezed by the increasing applied field, resulting in steeper $E_{\mathrm{VC}}(r)$ curves (see the solid colored lines). We present here three exemplary curves for nucleation fields for different meshes. Please note that a grid analysis is necessary only for the $E_{\mathrm{BP}}$ curves, as they contain the Bloch point and thus are strongly cell-size dependent [19]. We have extracted the switching field by examining the intersection of curves of $E_{\mathrm{VC}}+\Delta E_{\mathrm{ther}}$ and $E_{\mathrm{BP}}$ [see also the sample arrows in Fig. 7(b)]. Knowing the dependence $H_{\mathrm{sw}}(c)$, one can extrapolate it to zero mesh, similarly as earlier in the text [see the example for $300 \mathrm{~K}$ in Fig. 6 (right axis)]. 


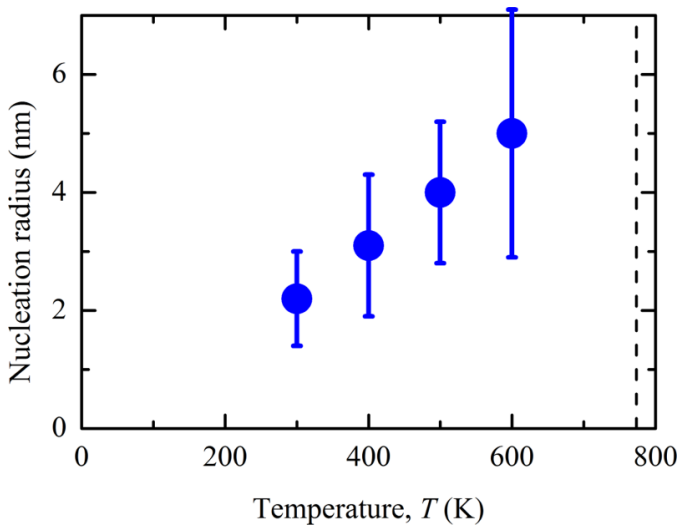

FIG. 8. (Color online) The radius of the nucleation half sphere.

The solid points in Fig. 3(a) summarize the results of our zero-mesh extrapolation procedure for all investigated temperatures. Results for low temperatures (absent in this figure) are difficult to analyze-here the necessary BP simulations would require smaller cell sizes. In contrast to the cell-size-dependent results (connected with dashed lines), now the results are almost linearly temperature dependent. Also, now they cross the horizontal axis at a temperature that differs remarkably from $T_{\mathrm{C}}$. We show in this figure results of both approaches, the "path method" (only for $H_{\mathrm{ext}}=0$ ) and the less precise "nucleation energy method." The latter suffers from the following simplifications: (i) the BP geometry does not change in the presence of $H_{\text {ext }}$, (ii) the BP geometry is not affected by the sample surface, (iii) the nucleation happens when the energy of the half sphere reaches a given value, and (iv) the dipolar interactions are neglected.

In Fig. 3(a) we show also experimental results for circular disks with radii 100 and $200 \mathrm{~nm}$ and thickness $50 \mathrm{~nm}$ (marked "Okuno" [4,5]) and for circular disks with $r=$ $500 \mathrm{~nm}$ and thickness $80 \mathrm{~nm}$ (marked "Kikuchi" [6]). The switching field does not significantly depend on $r$ and the dependence on the sample thickness is not known [5]. The wide range of experimental values is due to a variation of the behavior of different islands. In these experiments arrays of nominally identical islands were prepared but still, a large range of switching fields for different islands was observed. Additionally, the behavior of every single island, when observed several times, had its own distribution [4]. Thus, a comparison with experiments is in our case difficult. The main reason for the large difference between experimental data and our work is probably not the approximations of our approach but rather the fact that we have ignored the complex crystalline structure of the materials available in experiments. If instead of polycrystalline samples (permalloy) amorphous materials were investigated the observed distribution of $H_{\mathrm{sw}}$ was much narrower [5].

To support this statement we show in Fig. 8 the radius of the nucleation half sphere. This radius was retrieved by noting the position $r$ where the curves $E_{\mathrm{VC}}(r)$ and $E_{\mathrm{BP}}(r)$ cross; see the arrows in Fig. 7(b). Figure 8 can be compared with experimental data regarding crystallite size in permalloy. Its grains are usually much larger $[33,34]$. This leads us to the conclusion that precise simulations of quasistatic experiments must consider the given crystalline structure - the grain sizes as well as knowledge of the intergrain and intragrain exchange interactions.

\section{SUMMARY}

We have evaluated the switching conditions for a vortex for the case of slowly increasing orthogonal magnetic field applied antiparallel to the VC. The numerical problems related to VC reversal, caused by the singular character of the Bloch point, were avoided by applying a finite temperature. This approach allowed us to reliably model the $\mathrm{VC}$ behavior in a wide range of temperatures, an important improvement as compared to zero-temperature simulations presented in the literature so far. Additionally, we have performed an analysis where the influence of thermal excitation on crossing the energy barrier for BP nucleation was investigated. The switching field for room temperature is roughly $700 \mathrm{mT}$ in thin-film permalloy islands, then it drops almost linearly to zero for temperatures $100-200^{\circ} \mathrm{C}$ below the Curie point. The analysis of the nucleation volume revealed, however, that the situation in real samples is more complex. The crystalline structure of the evaluated material would have to be considered for an improved accuracy.

The presented approach is an important step towards a reliable modeling of Bloch points. We have shown that the inclusion of temperature makes the simulation more realistic and we have opened a path for future vortex core reversal simulations.

\section{ACKNOWLEDGMENTS}

The work was partially supported by the research Grant No. CZ.1.07/2.3.00/30.0055 of the Operational Programme Education for Competitiveness and co-financed by the European Social Fund and the state budget of the Czech Republic.
[1] A. Wachowiak, J. Wiebe, M. Bode, O. Pietzsch, M. Morgenstern, and R. Wiesendanger, Science 298, 577 (2002)

[2] K. Nakano, D. Chiba, N. Ohshima, S. Kasai, T. Sato, Y. Nakatani, K. Sekiguchi, K. Kobayashi, and T. Ono, Appl. Phys. Lett. 99, 262505 (2011).

[3] Q. Mistral, M. van Kampen, G. Hrkac, J.-V. Kim, T. Devolder, P. Crozat, C. Chappert, L. Lagae, and T. Schrefl, Phys. Rev. Lett. 100, 257201 (2008).
[4] A. Thiaville, J. M. Garcia, R. Dittrich, J. Miltat, and T. Schrefl, Phys. Rev. B 67, 094410 (2003).

[5] T. Okuno, K. Mibu, and T. Shinjo, J. Appl. Phys. 95, 3612 (2004).

[6] N. Kikuchi, S. Okamoto, O. Kitakami, Y. Shimada, S. G. Kim, Y. Otani, and K. Fukamichi, J. Appl. Phys. 90, 6548 (2001).

[7] R. Hertel, S. Gliga, M. Fahnle, and C. M. Schneider, Phys. Rev. Lett. 98, 117201 (2007). 
[8] Q. F. Xiao, J. Rudge, B. C. Choi, Y. K. Hong, and G. Donohoe, Appl. Phys. Lett. 89, 262507 (2006).

[9] M. Kammerer, M. Weigand, M. Curcic, M. Noske, M. Sproll, A. Vansteenkiste, B. Van Waeyenberge, H. Stoll, G. Woltersdorf, C. H. Back, and G. Schuetz, Nat. Commun. 2, 279 (2011).

[10] Y. Liu, S. Gliga, R. Hertel, and C. Schneider, Appl. Phys. Lett. 91, 112501 (2007).

[11] M.-W. Yoo, K.-S. Lee, D.-E. Jeong, and S.-K. Kim, Phys. Rev. B 82, 174437 (2010).

[12] K.-S. Lee, S.-K. Kim, Y.-S. Yu, Y.-S. Choi, K. Y. Guslienko, H. Jung, and P. Fischer, Phys. Rev. Lett. 101, 267206 (2008).

[13] B. Pigeau, G. de Loubens, O. Klein, A. Riegler, F. Lochner, G. Schmidt, and L. W. Molenkamp, Nat. Phys. 7, 26 (2011).

[14] B. Van Waeyenberge, A. Puzic, H. Stoll, K. W. Chou, T. Tyliszczak, R. Hertel, M. Fahnle, H. Bruckl, K. Rott, G. Reiss, I. Neudecker, D. Weiss, C. H. Back, and G. Schuetz, Nature (London) 444, 461 (2006).

[15] R. Hertel and H. Kronmüller, J. Magn. Magn. Mater. 238, 185 (2002).

[16] R. Hertel and J. Kirschner, J. Magn. Magn. Mater. 278, L291 (2004).

[17] J. Miltat and A. Thiaville, Science 298, 555 (2002).

[18] R. G. Elias and A. Verga, Eur. Phys. J. B 82, 159 (2011).

[19] K. M. Lebecki, D. Hinzke, U. Nowak, and O. ChubykaloFesenko, Phys. Rev. B 86, 094409 (2012).

[20] K. Vahaplar, A. M. Kalashnikova, A. V. Kimel, D. Hinzke, U. Nowak, R. Chantrell, A. Tsukamoto, A. Itoh, A. Kirilyuk, and T. Rasing, Phys. Rev. Lett. 103, 117201 (2009).

[21] B. C. Stipe, T. C. Strand, C. C. Poon, H. Balamane, T. D. Boone, J. A. Katine, J.-L. Li, V. Rawat, H. Nemoto, A. Hirotsune,
O. Hellwig, R. Ruiz, E. Dobisz, D. S. Kercher, N. Robertson, T. R. Albrecht, and B. D. Terris, Nat. Photon. 4, 484 (2010).

[22] S. Gliga, Y. Liu, and R. Hertel, J. Phys.: Conf. Ser. 303, 012005 (2011).

[23] D. A. Garanin, Phys. Rev. B 55, 3050 (1997).

[24] R. Skomski, Simple Models of Magnetism, Oxford Graduate Texts (Oxford University Press, New York, 2008).

[25] J. Miltat and M. J. Donahue, in Handbook of Magnetism and Advanced Magnetic Materials, edited by $\mathrm{H}$. Kronmüller and S. Parkin (Wiley-Interscience, Chichester, 2007), Vol. 2, pp. 742-764.

[26] K. M. Lebecki and U. Nowak, J. Appl. Phys. 113, 023906 (2013).

[27] D. Hinzke, N. Kazantseva, U. Nowak, O. N. Mryasov, P. Asselin, and R. W. Chantrell, Phys. Rev. B 77, 094407 (2008).

[28] E. G. Galkina, B. A. Ivanov, and V. A. Stephanovich, J. Magn. Magn. Mater. 118, 373 (1993).

[29] M. J. Donahue and D. G. Porter, oOMmF User's Guide, Tech. Rep. 6376 (NIST, Gaithersburg, 1999), http://math.nist.gov/oommf.

[30] N. Kazantseva, D. Hinzke, U. Nowak, R. W. Chantrell, U. Atxitia, and O. Chubykalo-Fesenko, Phys. Rev. B 77, 184428 (2008).

[31] R. Dittrich, T. Schrefl, D. Suess, W. Scholz, H. Forster, and J. Fidler, J. Magn. Magn. Mater. 250, L12 (2002).

[32] H. Kronmüller and M. Fähnle, Micromagnetism and the Microstructure of Ferromagnetic Solids (Cambridge University Press, Cambridge, UK, 2003).

[33] R. Gupta and M. Gupta, Phys. Rev. B 72, 024202 (2005).

[34] J. Fassbender, J. von Borany, A. Mucklich, K. Potzger, W. Moller, J. McCord, L. Schultz, and R. Mattheis, Phys. Rev. B 73, 184410 (2006). 